\title{
Psychometric Evaluation of Family Illness Perceptions of Relatives with Schizophrenia
}

\author{
Shih-Kai Lee ${ }^{1 *}$, Esther Ching-Lan Lin ${ }^{2 *, \dagger}$, Ya-Fen Chang ${ }^{3}$, Wen-Chuan Shao ${ }^{4}$, Ru-Band Lu ${ }^{5}$
}

\begin{abstract}
Background: Family caregivers in Taiwan significantly affect relatives with schizophrenia because Chinese culture values familial interdependence. A validated instrument is needed to examine the caregivers' perceptions of relatives with schizophrenia.
\end{abstract}

Purpose: This cross-sectional study tested the psychometric quality of the Illness Perception Questionnaire-Revised Version for Families of Patients with Schizophrenia (IPQ-RFS).

Methods: Two-hundred forty primary family caregivers of outpatients with schizophrenia living in a community in central Taiwan were purposively recruited. All participants completed the IPQ-RFS and the Perceived Psychiatric Stigma Scale (PPSS). The psychometric quality of the IPQ-RFS was evaluated using internal consistency and test-retest correlations for reliability and using correlations with PPSS and exploratory factor analysis for construct validity.

Results: Adequate Cronbach's a values showed internal consistencies, and significant correlations between 3 subscales and the 4-week test-retest results of the IPQ-RFS in most subscales validated the reliability of the IPQ-RFS subscales. Exploratory factor analysis showed good construct validity and correlations with PPSS: acceptable Kaiser-Meyer-Olkin measures of sampling adequacy, Bartlett's values, and factor loadings. Seven factors with 38 items explained $67.73 \%$ of the total variance.

Conclusion: We validated the psychometric quality of the IPQ-RFS. Future studies should investigate how family caregivers' perceptions of relatives with schizophrenia affect the treatment adherence and recovery of patients.

\section{Keywords}

Schizophrenia, Family, Illness Perception, Stigma, Reliability, Validity

\section{Introduction}

Because of the bidirectional and entangled relationships between patients and family systems, family critically affects the outcomes of relatives with schizophrenia. Specifically, the value of familial interdependence in Chinese traditional Confucian culture strongly affects illness adaptations and therapeutic strategies for relatives with mental illness [1].

Illness perception, defined as what patients believe

\footnotetext{
'Department of Nursing, Tsaotun Psychiatric Center, Ministry of Health and Welfare, Tsaotun Township, Nantou County 542, Taiwan ${ }^{2}$ Department of Nursing,College of Medicine, National Cheng Kung University (NCKU) and Hospital, Taiwan

${ }^{3}$ Department of Nursing, Tsaotun Psychiatric Center, Ministry of Health and Welfare, Tsaotun Township, Nantou County 542, Taiwan ${ }^{4}$ Dun Ren Psychiatric Hospital, Taiwan

${ }^{5}$ Department of Psychiatry, College of Medicine and Hospital, National Cheng-Kung University, Tainan, Taiwan

${ }^{\dagger}$ Author for correspondence: Esther Ching-Lan Lin, Department of Nursing, National Cheng Kung University (NCKU) and Hospital, 1 University Road, Tainan City 701, Taiwan

*The first two authors (Shih-Kai Lee and Esther Ching-Lan Lin) listed contributed equally to the study and received equal credit in this publication. Tel: 886-6-235-3535, 5038; email: chinglan@mail.ncku.edu.tw
} 
about their health problems, is closely correlated with a patient's adherence to treatment. What patients believe about their illness help them and their caregivers understand the illness [2]. Positive (understandable and controllable) beliefs, might help patients more actively manage their illness. Negative beliefs, however, might prevent this. In addition, evidence [3-5] supports the notion that specific family attitudes and family perceptions of a relative's mental illness are related to the patient's adherence to prescribed treatment.

The Common-Sense Model of Self-Regulation (CSM) [6] is a widely used theoretical framework for how individuals perceive their illnesses and how this subjective perception regulates individual emotional and behavioral responses to the illness and predicts health outcomes. It hypothesizes that individuals are problemsolvers who attempt to close the perceived gap between their current state of health and their future goals. It also hypothesizes that they will continuously appraise their outcome in relation to their desired state of health and modify their beliefs and behaviors accordingly, and that their self-regulation of coping strategies is guided by how they interpret their illness experiences. It includes two modes of illness representation: cognitive and affective modes. Using cognitive and emotional representations, this model also assumes that sociocultural and environmental factors contribute to patients' health beliefs and behaviors. The cognitive mode encompasses beliefs about (1) internal and external stimuli; (2) the treatment system; (3) representations of the illness and the treatment; (4) coping procedures; (5) evaluation of the cognitive treatment of the information; (6) emotional representation; (7) coping responses; (8) evaluation of the emotional treatment of the information. The Illness Perception Questionnaire (IPQ) [7] was developed to assess the key beliefs highlighted by the CSM. The IPQ-Revised (IPQ-R) [8] adds three subscales: "timeline cyclical" (how variable the symptoms are), "coherence" (how much individuals believe they understand their illness), and "emotional representation" (how much individuals experience emotional responses to illness). The emotional representation of the model provides a framework for exploring that is not mediated by a cognitive appraisal; however, there is insufficient evidence that this component exists. The IPQ-R was modified to suit specific research and illnesses, including practitioners [9], schizophrenia [10] and their family caregivers $[11,12]$. With small sample sizes, two studies
$[12,13]$ preliminary supported the psychometric quality of the IPQ-R in family caregivers with relatives with schizophrenia. A psychometrically attested and Chinese-version IPQ-R for family caregivers of patients with schizophrenia is still lacking.

How patients perceive their illness can predict how they manage the illness and what their health outcome will be [14-16]. Other studies $[10,17]$ have reported that how patients with schizophrenia perceive their illness affects their adherence to their medication regimen. Moreover, patients with schizophrenia and their family caregivers have discrepant perceptions of schizophrenia [5] and its consequences. This discrepancy is correlated with greater patient anxiety and depression, and lower patient self-esteem, and the discrepant views on the controllability of schizophrenia are associated with greater distress, depression, and lower self-esteem in family caregivers. In addition to having negative perceptions of schizophrenia, highly emotionally expressive family caregivers have perceptions discrepant to those of the relative with schizophrenia [13]. Depressed family caregivers are particularly pessimistic about their own persistence and controllability [5]. The discrepant views between patients and family caregivers about schizophrenia and its consequences correlate with greater anxiety and depression, and with lower self-esteem in patients, while the discrepant views on controllability correlate with greater distress, depression, and lower self-esteem in family caregivers. Therefore, a family member's explanations and perceptions of a relative's illness are associated with the family's expressed emotion and stigma, and then modify how the family and family caregiver cope with the patient's illness, distress, and burden.

In Chinese society, which is generally sociocentric, family can be an effective resource, and family caregivers are likely to be very involved in the patient's treatment, including accompanying patients to their clinic visits and staying with them (often 24 hours a day) while they are hospitalized $[18,19]$. The entire family typically shares the honor and bears the shame for all family members [20]. Chinese families might feel shameful and distressed because their mentally ill relative's symptomatic behaviors interfere with the cultural value of interpersonal harmony in Chinese culture [21,22]. Familial influence on the relative with schizophrenia might be modified by expressed emotion and family attitudes and perceptions of the illness, 
and, in turn, positively or negatively affect the outcome of the relative with schizophrenia. However, few studies have examined the effect of family perceptions of schizophrenia. Psychometrically attested instruments that assess the illness perception of patients with schizophrenia and of their family caregivers are needed.

\section{Methods}

\section{- Research design and setting}

This cross-sectional study was used in the outpatient department of an 1109-bed psychiatric center in central Taiwan to test the psychometric quality of the Chinese-version of the Illness Perception Questionnaires-Revised Version for Family of Patients with Schizophrenia (IPQRFS). The human rights protection protocols and all ethical considerations were approved by the hospital's Institutional Review Board (B-ER103-222) before any patients were recruited. Eligible participants were referred by psychiatrists and a research nurse explained all the study's details and informed them of their right to withdraw from the study at any time, and for any reason, in accordance with the Declaration of Helsinki. Signed written informed consents were obtained from all participants before data were collected by a research nurse either in the outpatient department or in participants' community.

\section{- Sample}

Two-hundred forty primary family caregivers of outpatients with schizophrenia living in the community were purposively recruited for this study. The primary family caregiver-usually the spouse, parent, sibling, or some other relativehad to be at least 20 years old and have lived with the patient for at least 1 year, and be able to read and understand Chinese. Caregivers diagnosed with a serious mental illness or mental retardation were excluded.

\section{- Instrument}

The sociodemographic data of the family caregiver include gender, age, education, marital status, and employment. Two self-rated instruments were used to assess how family caregivers perceive schizophrenia and psychiatric stigma.

\section{- Illness perception}

The Illness Perception Questionnaire-Revised
(IPQ-R) was developed based on the CSM model [8] (the Chinese version is provided on an IPQ-R website <http://www.uib.no/ipq/index. html>). The IPQ-R has three subscales. The first subscale, Identity, assesses illness symptoms using yes-no questions. The second subscale assesses 38 patient perceptions of illness: timeline (6 items), timeline cyclical (4 items), consequence (6 items), personal control (6 items), treatment control (5 items), illness coherence (5 items), and emotional representation (6 items). The third subscale has 18 patient perceptions of the cause of the illness: psychological attribution (6 items), risk factors (6 items), immunity (3 items), and accident (3 items). The second and third subscales are rated on a 5-point Likert scale ranging from 1 (strongly disagree) to 5 (strongly agree). Higher scores on identity, timeline, consequence, timeline cyclical, and emotional representation indicate patient beliefs about having more symptoms, chronicity of the condition, negative consequences of the illness, cyclical nature of the condition, and negative emotional representations. Higher scores on personal control, treatment control, and illness coherence indicate positive personal control, treatment control, and controllability of the illness. A higher score on the cause of illness indicates a stronger belief of illness attribution. The Chinese version of the IPQ-R for schizophrenia was tested in 210 patients with schizophrenia and found to have adequate internal consistency (Cronbach's $\bigotimes:$ 0.74-0.90) and adequate construct validity (acceptable Kaiser-Meyer-Olkin [KMO] values, Bartlett's values, and total sample variance 66.56\%) [23].

We transformed the Chinese version of the IPQ-R into the IPQ-RFS by changing the subject term in each item from "illness" to "schizophrenia" and slightly altering the wording so that the respondent is being asked about their "relative's illness" rather than their own. Because the first subscale (Identity) of the original IPQ-R mostly address on physical symptoms, the18-item Brief Psychiatric Rating Scale, related to psychotic symptoms in patients with schizophrenia, was used to assess the Identity subscale of the IPQRFS. The $17^{\text {th }}$ item was substituted into another 2 items assessed "avolition": don't want to do anything; and don't want to interact with anyone. The content validity of this subscale was supported by 5 clinical experts (the Content Validity Index $=0.88$ ). The psychometric quality of the IPQ-RFS was examined in 62 family caregivers with a relative diagnosed with 
schizophrenia [12], except for the Identity subscale. It has acceptable reliability (Cronbach's $\alpha: 0.71-0.84)$ and was associated with affiliate stigma. However, the entire instrument has not been tested with a sufficiently large sample.

\section{- Perceived Psychiatric Stigma}

The Perceived Psychiatric Stigma Scale (PPSS) [24] is a self-report scale that assesses the selfstigma of Chinese psychiatric patients. The 12 -item short version of the scale measures three factors: fear of social ostracism (4 items), concerns about marital preclusion (4 items), and self-deprecation ( 4 items). Ratings are based on a 4-point Likert scale ranging from "completely disagree (1)" to "completely agree (4)" (total score range: 12-48). Cronbach's $\alpha$ has been reported as 0.88 , and, after a 1 -week interval, test-retest reliability was 0.92 , and the perceived stigma of inpatients predicted low self-esteem was assessed [24,25]. We used the PPSS for relatives to test the associations with the IPQRFS as the convergent validity in this study.

\section{Statistical analysis}

Descriptive statistics of demographic variables are presented. The Cronbach's $\alpha$ and test-retest correlations of all the IPQ-RFS subscales for family were examined for internal consistency and stability of reliability. Correlations among 3 subscales of the IPQ-RFS and correlations with the PPSS, item analysis, and exploratory factor analysis (EFA) were used to determine construct validity. Participants were ranked based on IPQRFS scores and separated into two subgroups: the top and bottom $27 \%$. The two subgroups were then examined using an independent $t$ test for item discrimination, and then using EFA with principal component factor analysis with a varimax rotation. Significance was set at $\mathrm{p}<0.05$. SPSS 18.0 for Windows was used to analyze all data (SPSS Inc., Chicago, IL, USA).

\section{Results}

\section{- Family demographic characteristics}

Most family caregivers were female, married, employed, and had 6 years of education. Their mean age was $41.09 \pm 14.81$ (Table 1 ).

\section{- Family perceptions of schizophrenia}

The IPQ-RFS identity mean score was 7.91 \pm 5.30 (highest possible score $=19$ ), which indicated that families had not been adequately aware of their relatives' symptoms. It also indicated that relatives tended to attribute the illness to psychological factors, and perceived it as treatable, but chronic, cyclical, less personally controllable, and having negative consequences. The psychological attribution mean score was higher than the score of non-psychological factors $(2.74 \pm 0.70$ vs. $2.35 \pm 0.50)$ of the causes of schizophrenia, which indicated that families believed psychological factors to be its major causes. The mean of the family perceptions of schizophrenia was 3.23; the highest scores of the 4 items were timeline (3.60), consequence (3.48), treatment control (3.36), and emotional representation (3.32), which indicated that families' strong beliefs that the condition would endure (time-line), have negative consequences and emotional impact, and were confident that the condition could be managed by current treatment (Table 2).

\section{- Reliability of the IPQ-RFS}

Except for treatment control, the internal consistency of the IPQ-RFS reliability in families assessed using Cronbach's $\alpha$ (0.71-0.90) was appropriate (Table 2).

Significant correlations over the 4-week testretest of the IPQ-RFS subscales, ranged from $r=0.48$ to 0.68 (Table 2), which showed adequate associations (stability and reliability) between the two assessment points, except for illness perception, timeline, consequence, treatment control, and causes of illness subscales (Table 3).

\section{- Validity of the IPQ-RFS}

The acceptable correlation level of the dimensions of identity, illness perception, and attribution ranged from $r=0.24$ to 0.53 , which indicated that the three dimensions of illness perception were not redundant $(r<0.70)$ and that the discriminant validity was adequate [26] (Table 3). Significant correlations of the IPQ-RFS with the PPSS, except for psychological attribution, ranged from $r=0.14$ to 0.46 , which indicated adequate convergent validity.

Item analysis showed that the difference between the top and bottom $27 \%$ of all items was significant, which indicated good item discrimination. The KMO value of sampling adequacy was 0.82 and Bartlett's test of sphericity was 4144.02 ( $\mathrm{p}<0.001)$, which indicated that the IPQ-RFS met the assumption for EFA. The criteria for item selection from factor analysis included: (a) an eigenvalue > 1, (b) factor loadings $>0.3$, and (c) a minimum of three items 
for each factor. Seven factors of illness perception met these criteria and explained $67.73 \%$ of total sample variance, and the item structures of 7 factors were identical to the original item structure of the IPQ-R. Factor loading in each item ranged from 0.38 to 0.87 . Overall, the item analysis and EFA results indicated that the construct validity of IPQ-RFS was acceptable (Table 4).

\section{Discussion}

Our most important finding is that most of the IPQ-RFS-scales are reliable and valid. Our review of the literature showed that this is the first study with adequate sample size to present the family illness perception of schizophrenia in a Chinese society.

Most family caregivers attributed schizophrenia to psychological factors, and perceived it as treatable, but chronic, cyclical, less personally controllable than are most other illnesses, and having negative consequences. Having pessimistic beliefs often caused caregivers to have high levels of psychological distress [3], and might indicate their urgent need for knowledge about illness management and for emotional support.

The IPQ-RFS subscales showed overall satisfactory internal consistency in families of a relative with schizophrenia, except for treatment control (Cronbach's $\alpha=0.46$ ), but nonsignificant 4-week test-retest correlations were found in some subscales: illness perception (timeline, consequence, and treatment control) and causes of illness. A lower Cronbach's $\alpha$ level in treatment control may indicate the internal consistency of related items need to be improved. Nonsignificant 4-week test-retest correlations in some of illness perception and causes of illness subscales may reflect the time-related characteristics of these subscales, and future studies could longitudinally examine these subscales at different illness stages. Other studies have reported similar unsatisfactory internal consistency reliability in some aspects of illness perception. One [27] reported that, for the IPQ-R in German-speaking outpatients with chronic schizophrenia, the personal control and illness coherence subscales (Cronbach's $\alpha$ : 0.52, 0.48 ) did not have acceptable levels of internal consistency $(>0.70)$, but test-retest correlations

\begin{tabular}{|c|c|}
\hline Variables & Values \\
\hline Age (years) & $41.09 \pm 14.81$ \\
\hline \multicolumn{2}{|l|}{ Gender } \\
\hline Male & $107(45.0)$ \\
\hline Female & $131(55.0)$ \\
\hline \multicolumn{2}{|l|}{ Educational Level } \\
\hline Illiterate & $15(6.3)$ \\
\hline Elementary school & $134(55.8)$ \\
\hline Junior high school & $37(15.4)$ \\
\hline Senior high school & $37(15.4)$ \\
\hline Four-year college & $16(6.7)$ \\
\hline Master's degree & $1(0.4)$ \\
\hline \multicolumn{2}{|l|}{ Marital status } \\
\hline Married & 207 (86.2) \\
\hline Unmarried & 33 (13.8) \\
\hline \multicolumn{2}{|l|}{ Employment } \\
\hline Employed & $150(60.7)$ \\
\hline Unemployed & $90(39.3)$ \\
\hline
\end{tabular}

\begin{tabular}{|c|c|c|c|}
\hline Variables (Scores) & Values & $a$ & Test-retest $(\mathbf{r})^{\mathrm{a}}$ \\
\hline Identity & & 0.90 & $0.64^{* *}$ \\
\hline Symptom awareness & $0.49 \pm 0.26$ & 0.86 & $0.68^{* *}$ \\
\hline Symptom attribution & $0.38 \pm 0.27$ & 0.90 & $0.64^{* *}$ \\
\hline IIIness Perception & & 0.75 & -0.05 \\
\hline Timeline & $3.60 \pm 0.68$ & 0.82 & 0.28 \\
\hline Consequence & $3.48 \pm 0.71$ & 0.83 & 0.40 \\
\hline Personal control & $3.12 \pm 0.47$ & 0.71 & $0.56^{* *}$ \\
\hline Treatment control & $3.36 \pm 0.46$ & 0.46 & 0.40 \\
\hline IIIness coherence & $2.60 \pm 0.64$ & 0.81 & $0.49^{*}$ \\
\hline Timeline cyclical & $2.99 \pm 0.72$ & 0.81 & $0.48^{*}$ \\
\hline Emotional representation & $3.32 \pm 0.71$ & 0.82 & $0.67^{* *}$ \\
\hline Causes of illness & & 0.78 & 0.41 \\
\hline Psychological attribution & $2.74 \pm 0.70$ & 0.75 & 0.42 \\
\hline Non-psychological attribution & $2.35 \pm 0.50$ & 0.72 & 0.26 \\
\hline \multicolumn{4}{|c|}{$\begin{array}{l}\text { All values are mean } \pm \text { standard deviation unless otherwise indicated. } \\
\text { Pearson's correlation was used to examine the 4-week test-retest reliability of the IPQ-RFS subscales in } 20 \text { participants. } \\
{ }^{*} \mathrm{p}<0.05 ;{ }^{* *} \mathrm{p}<0.01 \text {. }\end{array}$} \\
\hline
\end{tabular}


Research Shih-Kai Lee \& Esther Ching-Lan Lin

\begin{tabular}{|c|c|c|c|c|c|c|c|c|c|c|c|c|c|c|}
\hline & \multirow[t]{2}{*}{ Identity } & \multicolumn{2}{|l|}{ Identity } & \multirow{2}{*}{\begin{tabular}{|l|} 
Illness \\
Percep
\end{tabular}} & \multirow[t]{2}{*}{$\begin{array}{l}\text { Time } \\
\text { line }\end{array}$} & \multirow[t]{2}{*}{ Conseq } & \multirow{2}{*}{\begin{tabular}{|l|} 
Personal \\
Control
\end{tabular}} & \multirow{2}{*}{\begin{tabular}{|l|} 
Treatment \\
Control
\end{tabular}} & \multirow{2}{*}{$\begin{array}{l}\text { Illness } \\
\text { Coherence }\end{array}$} & \multirow{2}{*}{\begin{tabular}{|l|}
$\begin{array}{l}\text { Time } \\
\text { line }\end{array}$ \\
Cyclical \\
\end{tabular}} & \multirow{2}{*}{$\begin{array}{l}\text { Emotional } \\
\text { Rep }\end{array}$} & \multirow{2}{*}{\begin{tabular}{|l|} 
IIIness \\
Causes
\end{tabular}} & \multirow{2}{*}{\begin{tabular}{|l|} 
Psych \\
Attrib
\end{tabular}} & \multirow{2}{*}{$\begin{array}{l}\text { Non- } \\
\text { psych } \\
\text { Factors }\end{array}$} \\
\hline & & Aware & Attrib & & & & & & & & & & & \\
\hline \multicolumn{15}{|c|}{ ( } \\
\hline \multicolumn{15}{|l|}{ Awareness } \\
\hline Attribution & & $0.777^{* *}$ & & & & & & & & & & & & \\
\hline $\begin{array}{l}\text { Illness } \\
\text { perception }\end{array}$ & $0.525^{* *}$ & $0.516^{* *}$ & $0.492^{* *}$ & & & & & & & & & & & \\
\hline Timeline & & $0.308^{* *}$ & $0.185^{* * *}$ & $0.598^{* *}$ & & & & & & & & & & \\
\hline Consequence & & $0.503^{* *}$ & $0.520^{* *}$ & $0.748^{* * *}$ & $0.496^{* *}$ & & & & & & & & & \\
\hline Personal & & -0.015 & -0.019 & 0.055 & $-0.253^{* *}$ & $-0.278^{* * *}$ & & & & & & & & \\
\hline \multicolumn{15}{|l|}{ control } \\
\hline Treatment & & $-0.189^{* *}$ & $-0.203^{* * *}$ & 0.066 & 0.024 & $-0.213^{* *}$ & $0.269^{* *}$ & & & & & & & \\
\hline \multicolumn{15}{|l|}{ control } \\
\hline Illness & & -0.013 & 0.030 & $0.129^{*}$ & $-0.151^{*}$ & -0.077 & $0.146^{*}$ & 0.114 & & & & & & \\
\hline \multicolumn{15}{|l|}{ coherence } \\
\hline Timeline & & $0.288^{* *}$ & $0.323^{* *}$ & $0.539^{* *}$ & $0.141^{*}$ & $0.411^{* *}$ & $-0.219^{* * *}$ & $-0.271^{* *}$ & $-0.138^{*}$ & & & & & \\
\hline \multicolumn{15}{|l|}{ cyclical } \\
\hline Emotional & & $0.418^{* *}$ & $0.408^{* *}$ & $0.638^{* *}$ & $0.206^{* *}$ & $0.466^{* *}$ & -0.110 & $-0.267^{* *}$ & $-0.274^{* *}$ & $0.516^{* *}$ & & & & \\
\hline \multicolumn{15}{|l|}{ representation } \\
\hline $\begin{array}{l}\text { Causes of } \\
\text { illness }\end{array}$ & $0.239 * *$ & $0.156^{*}$ & 0.117 & $0.217^{* * *}$ & $0.177^{* *}$ & $0.173^{* *}$ & $0.170^{* *}$ & -0.018 & $-0.246^{* *}$ & 0.092 & $0.221^{* *}$ & & & \\
\hline Psychological & & 0.082 & 0.083 & 0.101 & $0.152^{*}$ & 0.114 & 0.127 & 0.045 & $-0.199^{* *}$ & -0.087 & 0.095 & $0.737^{* * *}$ & & \\
\hline $\begin{array}{l}\text { Non- } \\
\text { psychological }\end{array}$ & & $0.164^{*}$ & 0.108 & $0.243^{* * *}$ & $0.146^{*}$ & $0.161^{*}$ & $0.156^{*}$ & -0.051 & $-0.198^{* *}$ & $0.194^{* *}$ & $0.247^{* *}$ & $0.881^{* *}$ & & \\
\hline PPSS & & $0.270^{* *}$ & $0.248^{* *}$ & $0.334^{* *}$ & $0.200^{* *}$ & $0.313^{* *}$ & $-0.219^{* * *}$ & $-0.142^{*}$ & $-0.242^{* *}$ & $0.396^{* *}$ & $0.462^{* *}$ & $0.183^{* *}$ & -0.007 & $0.263^{* *}$ \\
\hline
\end{tabular}

in all subscales significant. Two others $[9,28]$ also reported low Cronbach $\alpha$ values in treatment control and personal control in a test of the IPQ-R for healthcare professionals. Another two $[3,12]$, however, reported satisfactory internal reliability for all IPQ-R subscales in a test for caregivers of relatives with schizophrenia.

Three IPQ-RFS dimensions and the PPSS item discrimination, discriminant validity, and convergent validity were adequate based on significant differences of item analysis and acceptable correlation levels. Seven factors of illness perception, which adequately explained the variance and acceptable factor loading in each item were identical to the original item structures in the IPQ-R. Overall, the psychometric quality of the IPQ-RFS was adequate.

Another two studies [12,13], used the 58-item Identity subscale, also tested the psychometric quality of IPQ-R in family caregivers of a relative with schizophrenia. Considering of clinical implication in the future, the IPQ-RFS of our study adapted a 19-item Identity subscale from the BPRS. Except for Identity subscale, our study and these 2 studies used the same IPQ-R subscales. An earlier study [13] supported the concurrent validity of the modified IPQ in 47 family caregivers of relatives with schizophrenia with significant associations between caregivers' functioning and the consequences IPQ subscales and suitable inter-subscale correlations. A Taiwanese study [12] also supported the psychometric quality of the IPQ-RFS in 62 family caregivers with a relative diagnosed with schizophrenia. It reported acceptable reliability and an association with affiliate stigma. The present study validated the psychometric quality of the IPQ-RFS and we expect that it will provide a way to examine the factors related to family illness perception and to develop interventions that will improve constructive beliefs and supportive caregiving.

This study has two limitations. First, the study sample, family primary caregivers from the outpatient department of a psychiatric center in central Taiwan, might not be representative of all relatives of patients with schizophrenia in other Chinese-speaking populations. Second, the validity testing of the IPQ-RFS in the present study was limited to the internal consistency reliability and construct validity and used only item analysis and EFA. We suggest that future studies test different aspects of reliability and validity. For example, test-retest reliability and pre- 


\begin{tabular}{|c|c|c|c|}
\hline Item & Factor loading & Eigenvalue & Variance explained \\
\hline Factor 1. Timeline & & 3.38 & 10.25 \\
\hline IP1 & 0.72 & & \\
\hline IP2 & 0.79 & & \\
\hline IP3 & 0.68 & & \\
\hline IP4 & 0.79 & & \\
\hline IP5 & 0.77 & & \\
\hline IP18 & 0.72 & & \\
\hline Factor 2. Consequence & & 2.74 & 7.19 \\
\hline IP6 & 0.42 & & \\
\hline IP7 & 0.78 & & \\
\hline IP8 & 0.62 & & \\
\hline IP9 & 0.73 & & \\
\hline IP10 & 0.72 & & \\
\hline IP11 & 0.64 & & \\
\hline Factor 3. Personal control & & 2.46 & 9.75 \\
\hline IP12 & 0.41 & & \\
\hline IP13 & 0.86 & & \\
\hline IP14 & 0.87 & & \\
\hline IP15 & 0.68 & & \\
\hline IP16 & 0.81 & & \\
\hline IP17 & 0.71 & & \\
\hline Factor 4. Treatment control & & 3.10 & 10.22 \\
\hline IP19 & 0.54 & & \\
\hline IP20 & 0.71 & & \\
\hline IP21 & 0.83 & & \\
\hline IP22 & 0.56 & & \\
\hline IP23 & 0.55 & & \\
\hline Factor 5. Illness coherence & & 4.11 & 12.01 \\
\hline IP24 & 0.77 & & \\
\hline IP25 & 0.83 & & \\
\hline IP26 & 0.85 & & \\
\hline IP27 & 0.85 & & \\
\hline IP28 & 0.64 & & \\
\hline Factor 6. Timeline cyclical & & 1.28 & 6.13 \\
\hline IP29 & 0.69 & & \\
\hline IP30 & 0.77 & & \\
\hline IP31 & 0.68 & & \\
\hline IP32 & 0.68 & & \\
\hline Factor 7. Emotional rep & & 8.22 & 11.02 \\
\hline IP33 & 0.73 & & \\
\hline IP34 & 0.82 & & \\
\hline IP35 & 0.46 & & \\
\hline IP36 & 0.38 & & \\
\hline IP37 & 0.77 & & \\
\hline IP38 & 0.69 & & \\
\hline Total variance explained & & & $67.73 \%$ \\
\hline
\end{tabular}

dictive validity should be examined in longitudinal studies. We also recommend rigorous testing methods like the multitrait-multimethod.

\section{Conclusion}

Family caregivers significantly affect their relatives with schizophrenia in Taiwan. The psychometric quality of the Illness Perception Questionnaire-Revised Version for Families of Patients with Schizophrenia (IPQ-RFS) was validated. We expect that future studies can examine the factors related to family illness perception and investigate how family 
caregivers' illness perceptions affect their illness management and the recovery of patients with schizophrenia.

\section{Acknowledgements}

This research was funded by grant \#10553 from the Tsaotun Psychiatric Center, Ministry of Health and Welfare, MOST104-2314-B-006-047-MY3 from Ministry of Science and Technology and \#A1031 from E-DA Hospital.

\section{References}

1. Yen WJ, Teng $\mathrm{CH}$, Huang $\mathrm{XY}$, et al. A theory of meaning of caregiving for parents of mentally ill children in Taiwan, a qualitative study. J. Clin. Nurs. 19(1-2), 259-265 (2010).

2. Wright LM, Bell JM. Beliefs and IIIness: $A$ Model for Healing. [Calgary]: 4th Floor Press (2009).

3. Fortune DG, Smith JV, Garvey K. Perceptions of psychosis, coping, appraisals, and psychological distress in the relatives of patients with schizophrenia: An exploration using self-regulation theory. Br. J. Clin. Psychol. 44, 319-331 (2005).

4. Glick ID, Stekoll AH, Hays S. The role of the family and improvement in treatment maintenance, adherence, and outcome for schizophrenia. J. Clin. Psychopharmacol 31(1), 82-85 (2011).

5. Lobban F, Barrowclough C, Jones S. Does expressed emotion need to be understood within a more systemic framework? An examination of discrepancies in appraisals between patients diagnosed with schizophrenia and their relatives. Soc. Psychiatry Psychiatr. Epidemiol 41, 50-55 (2006).

6. Leventhal H, Phillips LA, Burns E. The Common-Sense Model of Self-Regulation (CSM): A dynamic framework for understanding illness self-management. J. Behav. Med 39(6), 935-946 (2016).

7. Weinman J, Petrie KJ, Moss-Morris $\mathrm{R}$, et al. The illness perception questionnaire: A new method for assessing the cognitive representation of illness. Psychol. Health 11(3), 431445 (1996).

8. Moss-Morris, R, Weinman J, Petrie K, et al. The Revised Illness Perception Questionnaire (IPQ-R). Psychol. Health 17(1), 1-16 (2002).

9. Arat $S$, Van den Zegel A, Van Rillaer M, et al. Development and preliminary evaluation of the validity and reliability of a revised illness perception questionnaire for healthcare

professionals. BMC Nurs 15, 34 (2016).

10.Lobban F, Barrowclough C, Jones S. Assessing cognitive representations of mental health problems. I. The illness perception questionnaire for schizophrenia. Br. J. Clin. Psychol 44(Pt 2), 147-162 (2005a).

11.Lobban, F, Barrowclough C, Jones S. Assessing cognitive representations of mental health problems. II. The illness perception questionnaire for schizophrenia: Relatives' version. Br. J. Clin. Psychol 44(Pt 2), 163-179 (2005b).

12.Huang $\mathrm{CH}$, Li SM, Shu BC. Exploring the relationship between illness perceptions and negative emotions in relatives of people with schizophrenia within the context of an affiliate stigma model. J. Nurs. Res 24(3), 217223 (2016).

13.Barrowclough C, Lobban F, Hatton $C$, et al. Investigation of models of illness in carers of schizophrenic patients using the Illness Perception Questionnaire. Br. J. Clin. Psychol 40, 371-385 (2001).

14.Dempster M, Howell D, McCorry NK. Illness perceptions and coping in physical health conditions: A metaanalysis. J. Psychosom. Res 79(6), 506513 (2015).

15. Hudson JL, Bundy C, Coventry PA, et al. Exploring the relationship between cognitive illness representations and poor emotional health and their combined association with diabetes self-care: A systematic review with meta-analysis. J. Psychosom. Res 76(4), 265-274 (2014).

16.Jaremo P, Arman $M$, Gerdle B, et al. Illness beliefs among patients with chronic widespread pain - associations with self-reported health status, anxiety and depressive symptoms and impact of pain. BMC Psychol 5(1), 24 (2017).

17.Rungruangsiripan $M$, Sitthimongkol $Y$, Maneesriwongul W, et al. Mediating role of illness representation among social support, therapeutic alliance, experience of medication side effects, and medication adherence in persons with schizophrenia. Arch. Psychiatr. Nurs 25(4), 269-283 (2011).

18. Huang $X Y$, Hung BJ, Sun FK, et al. The experiences of carers in Taiwanese culture who have longterm schizophrenia in their families: A phenomenological study. J. Psychiatr. Ment. Health Nurs 16(10), 874-883 (2009).

19.Kung WW. The illness, stigma, culture, or immigration? Burdens on Chinese American caregivers of patients with schizophrenia. Fam. Soc. 84, 547-557 (2003).

20.Sue S, Morishima JK. The Mental Health of Asian Americans. San Francisco, US: Jossey-Bass (1982).

21.Cheung F. Psychopathology among Chinese people. In Bond MH (ed.). The Psychology of Chinese People. Hong Kong, China: Oxford University Press, pp. 171-212 (1986).

22.Gabrenya WK, Hwang KK. Chinese social interaction: harmony and hierarchy on the good earth. In Bond $\mathrm{MH}$ (ed.). The Handbook of Chinese Psychology Hong Kong, China: Oxford University Press, pp. 309-321 (1996).

23.Shao WC, Lin ECL. A Survey of Medication Adherence and Related Factors among Patients with Schizophrenia Who Received Home Care Service Taipei: Final Report of Research Development Grant, Affiliate Hospitals of Department of Health, Executive Yuan (2011).

24.Han DY, Chen SH. Psychometric properties of the perceived psychiatric stigma scale and its short version. Formos. J. Ment. Health 21(3), 273-290 (2008).

25.Tseng CJ, Chiou JY, Yen WJ, et al. Associations between stigma perception and stigma coping behavior and quality of life in 
schizophrenic patients treated at a community rehabilitation center. J. Nurs 59(4), 62-70 (2012).

26.Cohen J. Statistical Power Analysis for the Behavioral Sciences (2nd ed.). Hillsdale, $\mathrm{NJ}$ : Lawrence Earlbaum Associates (1988).
27.Cavelti M, Contin G, Beck EM, et al. Validation of the IIIness Perception Questionnaire for Schizophrenia in a German-speaking sample of outpatients with chronic schizophrenia. Psychopathology 45(4), 259-269(2012).

28.Martin C, Fleming M, McCorkindale
S. Measurement properties of the Illness Perception Questionnaire in practitioners working with patients with co-morbid schizophrenia and learning disability. Eur. J. Pers. Cent. Healthc 4(1), 215-220 (2016). 